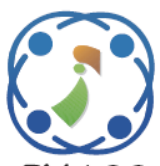

\title{
An Effective Temperature Monitoring Scheme Using ANN-Based Fiber Bragg Grating Sensor
}

\author{
Marwin Kannamplavil Mathew ${ }^{1}$ \\ ${ }^{1}$ School of Electronics Engineering, VIT University, Vellore, India \\ * Corresponding author's Email: vegenaveen@vit.ac.in
}

Rahul George John Valooran ${ }^{1}$ Naveen Kumar Vaegae ${ }^{1 *}$

\begin{abstract}
In this paper, a fiber Bragg grating (FBG) based temperature sensor is designed and implemented using a conventional laser source. It is observed that the sensitivity of FBG temperature sensor is low and not effective in sensing temperature of critical applications. Hence, FBG temperature sensitization encapsulation technique is introduced to increase the sensitivity of the sensor. The sensitivity is improved by a factor of 44 with temperature sensitization encapsulation technique, but the linearity error is $\pm 15 \%$ full scale reading. In order to retain the high sensitivity without compromising the linearity of the system, multilayer perceptron (MLP) artificial neural network (ANN) is introduced to estimate and reduce the linearity error of FBG sensor. The Levenberg-Marquardt (LM) algorithm is used for training and learning mechanism of MLP-ANN. A linearity error of approximately $\pm 2.8 \%$ is achieved without affecting the sensitivity of the system.
\end{abstract}

Keywords: Fiber Bragg grating, Neural networks, Temperature sensitization encapsulation, Thermal expansion.

\section{Introduction}

All in the past, the standard instrument to quantify physical and mechanical marvels is by utilizing electrical sensors. Regardless of their universality, these sensors have natural impediments, for example, transmission misfortune and weakness to electromagnetic obstruction, which makes their utilization illogical in numerous applications. Different strategies and arrangements have been examined to take care of these issues, and various approaches have been proposed to change the electrical medium to light medium. Light has a great deal of points of interest because of its high speed and as it's transmitted through optical fiber as a medium.

The innovation and uses of optical fibers has advanced quickly as of late. One of their applications is the fiber optic sensor which is initially developed for scientific purposes [1]. However now, this fiber optic sensor can be added to the business esteem. One working rule of fiber optic sensor that is utilized as a part of this venture is utilization of FBG sensors to gauge physical changes, for example, strain, temperature and pressure [2]. This sensor has phenomenal application esteem, particularly in the territory of disseminated implanted detecting in materials for utilizing on smart structures, for example bridges, aircrafts, pipelines and dams.

There are a wide range of fiber optic sensors working on various standards such as intensity modulation, polarization impacts, interferometry, refractive index changes, reflectometry etc. One such moderately developed type of fiber optic sensor which has all the earmarks of being especially alluring in numerous applications is the FBG [3]. There are various kinds of FBGs such as uniform, apodized, pi-phase shifted [4] and each has different sensitivity and bandwidth. In this paper, a uniform FBG sensor, which has a constant grating period is used and is considered to be excellent strain and temperature sensing device [5-7].

In general, temperature sensors require high sensitivity, linearity and stability [8-10]. Since bare uniform FBG temperature sensors have less sensitivity which hinders us from using them for 
critical applications $[11,12]$. In this paper, temperature sensitization encapsulation technique is introduced to increase the sensitivity of FBG sensor. However, this technique has its own limitations such as it reduces the linearity which is a vital factor for practical sensing applications [13]. In order to utilize the high sensitivity factor without compromising on the linearity, MLP-ANN technique is implemented to estimate and reduce the linearity error of the sensor. The ANN technique is mainly preferred as it requires fewer operations as compared to conventional numerical methods and the operations do not increase even if the number of calibration points increases [14].

The rest of the paper is organized as follows. Section 2 describes the methodology encompassing FBG sensor, temperature sensitization and MLPANN. Section 3 discusses the simulation results and conclusions are presented in section 4 .

\section{Methodology}

The proposed temperature measurement scheme consists of two stages and is shown in Fig. 1. The first stage consists of temperature measurement by FBG sensor and temperature sensitization encapsulation process. Even though the temperature sensitization encapsulation process enhances the sensitivity, the linearity is highly reduced. The MLP-ANN trained with LM-algorithm is used in the second stage to reduce the linearity error of the FBG sensor with temperature sensitization encapsulation to an appreciable range.

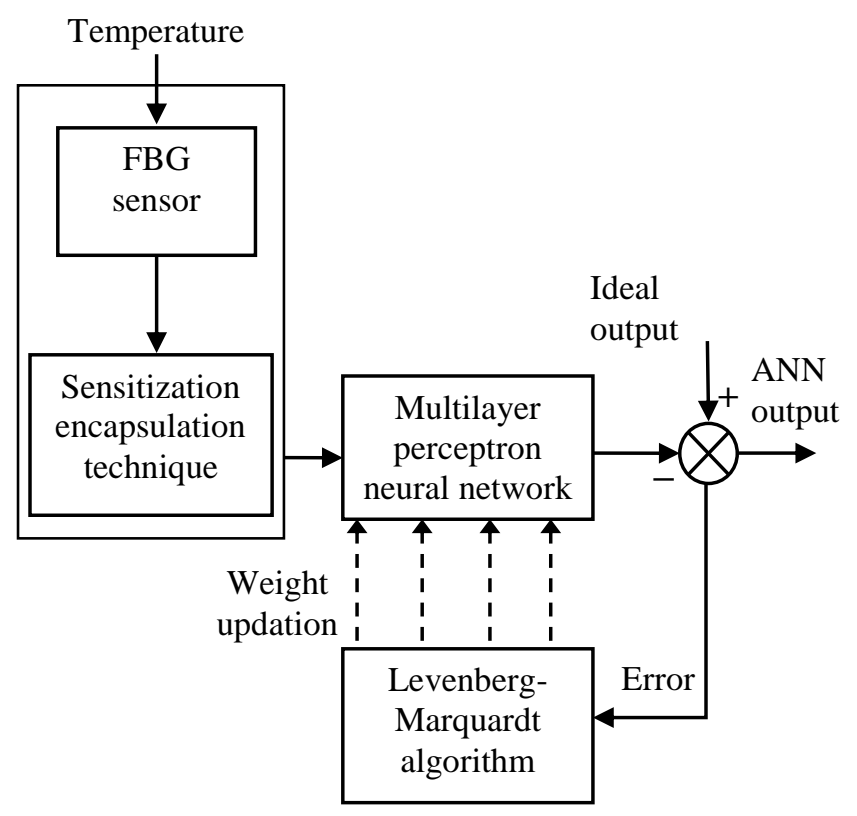

Figure.1 Block diagram of the proposed technique

\subsection{Uniform FBG}

A Fiber Bragg Grating (FBG) is a sort of disseminated Bragg reflector developed by varying refractive indices in a short portion of optical fiber that reflects specific wavelengths of light and transmits all others. A basic block diagram of a uniform FBG system is shown in Fig. 2.

At the point when a wide spectrum beam of light is sent to a FBG, reflections from each fragment of the varying refractive index in the grating region meddle helpfully just for a particular wavelength of light, called the Bragg wavelength $\left(\lambda_{b}\right)$ as observed in Eq. (1). This viably uses the FBG to mirror a particular frequency of light while transmitting all others. The Bragg wavelength $\left(\lambda_{b}\right)$ is an element of the spacing between the gratings $(\Lambda)$ and the effective refractive index $\left(n_{e}\right)$ of the fiber core.

The equations that govern FBG are as follows:

$$
\begin{aligned}
& \lambda_{b}=2 n_{e} \Lambda \\
& \Delta \lambda_{b}=\lambda(1-\rho \alpha) \Delta \varepsilon+\lambda_{b}(\alpha+\xi) \Delta T
\end{aligned}
$$

where $\Delta \lambda_{b}$ is the change in Bragg wavelength, $\rho \alpha, \alpha$ and $\xi$ are respectively the photo elastic, thermal expansion and thermo optic coefficients of the fiber, $\Delta \varepsilon$ is the change of strain and $\Delta T$ is the temperature change.

When the temperature of the fiber changes $(\Delta T)$, this will in turn cause the Bragg wavelength to change $\left(\Delta \lambda_{b}\right)$ as seen in Eq. (2) and this dependence of temperature on wavelength leads to design and implement a temperature monitoring system.

\subsection{Normal uniform FBG sensor using OptSim}

The framework for the FBG sensor is implemented in OptSim version 5.2 and is shown in Fig. 3(a). The transmitter section comprises of a pseudo-random binary sequence (PRBS) generator, an electrical generator, an electrical filter, an external modulator and $\mathrm{CW}$ laser. The function of the transmitter is to create a light source that passes

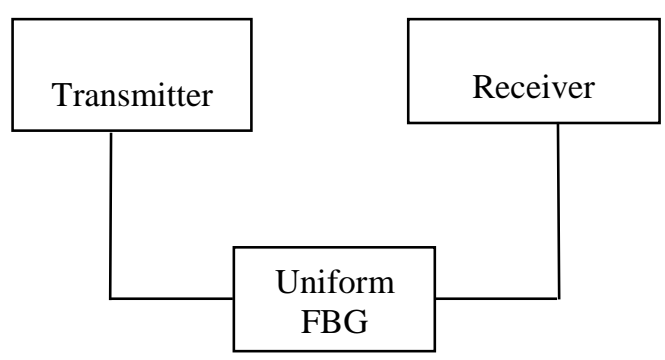

Figure.2 Block diagram of uniform FBG system 
through the FBG and whose output parameters are measured. Next, in the model it additionally has a fiber optic as transmission medium which consists of the Fiber Bragg Grating. The receiver section consists of receiver one and receiver two. The optical detector in this model is installed in the receiver part. A detector's capacity is to change over the received optical signal into an electrical signal, which is then amplified. The normal uniform FBG sensor measures the temperature in the range of $20^{\circ} \mathrm{C}-320^{\circ} \mathrm{C}$ with good linearity, but the temperature sensitivity is $0.375 \mathrm{pm} /{ }^{\circ} \mathrm{C}$, which makes the sensor unsuitable for critical sensing applications. So temperature sensitization encapsulation technique is required.

\subsection{Temperature sensitization encapsulation technique using OptiSystem}

The temperature sensitivity of the FBG setup shown in Fig. 3(a) is $0.375 \mathrm{pm} /{ }^{\circ} \mathrm{C}$ which is too low for sensing applications, so temperature sensitization encapsulation technique is adapted.
The temperature sensitization encapsulation technique scheme is implemented using OptiSystem version 14.2 as it has the capability to vary the thermal expansion coefficient which is vital for executing this scheme. This technique is used to provide the FBG encased in the substrate material with high thermal expansion $(\alpha s>>\alpha)$. The thermal expansion coefficient of typically utilized materials is shown in Table 1. The thermal expansion coefficient of fiber is just $0.5 \times 10^{-6} /{ }^{\circ} \mathrm{C}$ and is very low compared to Teflon and epoxy. Therefore, the temperature sensitivity of the FBG setup shown in Fig. 3(a) is improved by designing a circuit in OptiSystem and increasing the value of thermal expansion coefficient $(\alpha)$ to $40 \times 10^{-6} /{ }^{\circ} \mathrm{C}$. The circuit designed in OptiSystem is shown in Fig. 3(b). As described in Eq. (2), when there is an increase in the value of thermal expansion coefficient $(\alpha)$, there is a greater shift in the wavelength and hence improves the sensitivity of the sensor system.

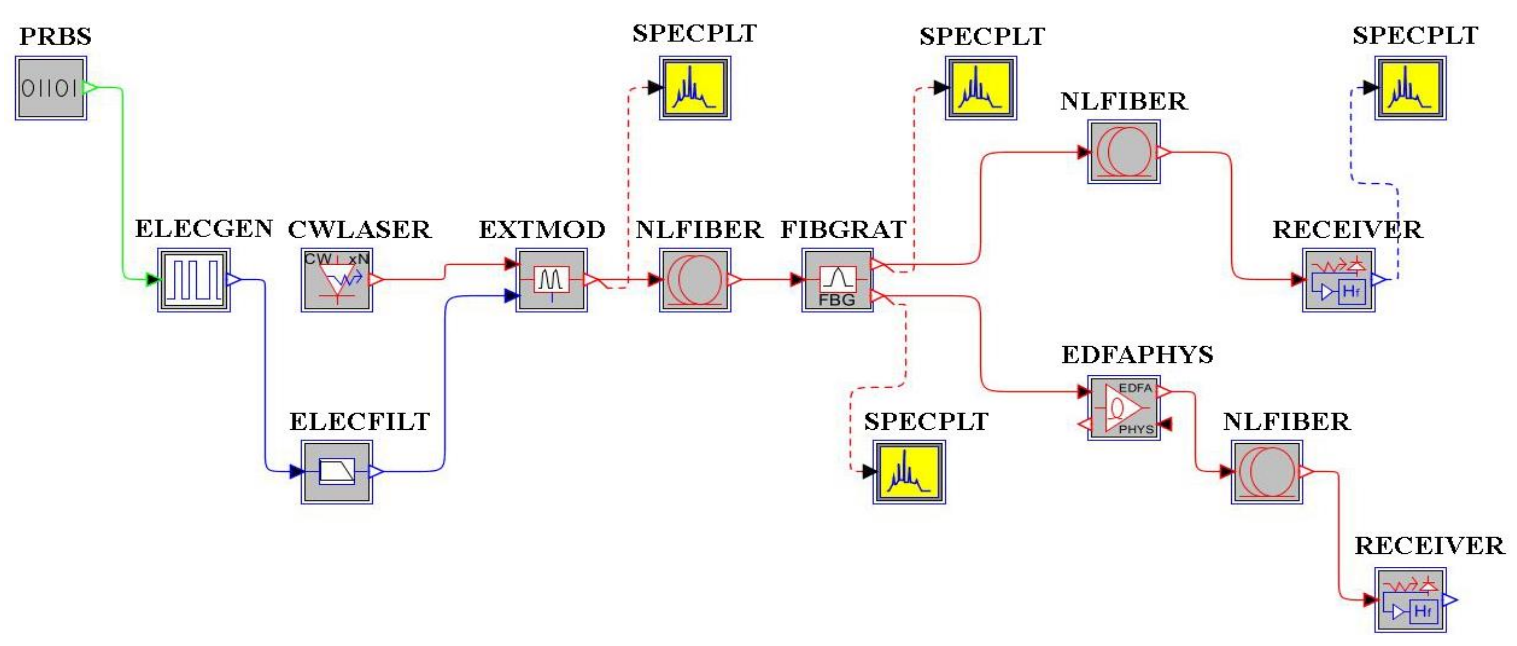

(a)

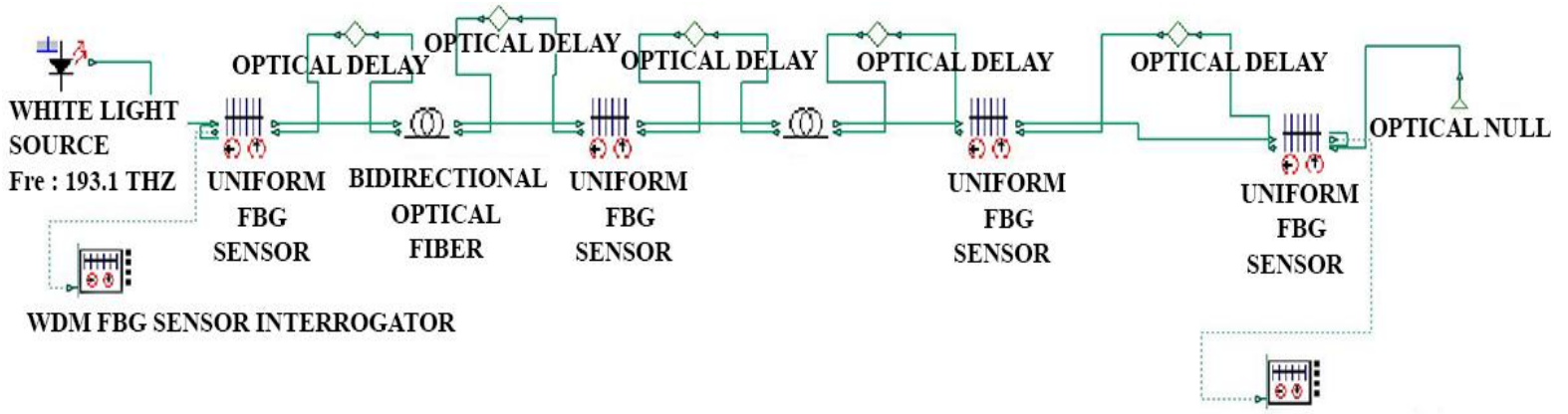

WDM FBG SENSOR INTERROGATOR

(b)

Figure.3 Simulation circuit design of FBG sensor: (a) Temperature measurement setup in OptSim and (b) Temperature sensitization encapsulation setup in OptiSystem 
Table 1. Thermal expansion coefficient

\begin{tabular}{|c|c|c|c|c|}
\hline Material & Fiber & $\begin{array}{c}\text { Metal } \\
\text { pipe }\end{array}$ & Teflon & Epoxy \\
\hline $\begin{array}{c}\text { Thermal } \\
\text { expansion } \\
\text { coefficient } \\
\left(10^{-6} / \mathrm{K}\right)\end{array}$ & 0.5 & 11.7 & $70-100$ & $40-80$ \\
\hline
\end{tabular}

\subsection{Artificial neural network technique}

In order to utilize the high sensitivity factor achieved by using temperature sensitization encapsulation technique and preserve the linearity, MLP-ANN technique is employed [14]. A basic MLP-ANN is shown in Fig. 4.

The MLP-ANN has three neurons in the hidden layer and one neuron in the input and output layers each. The log sigmoid and linear activation functions are utilized as a part of the hidden and output layer activation functions respectively. The system is trained by the LM-algorithm. The LMalgorithm is the most effective algorithm for small and medium measurement systems, as it has a faster converging time and least mean square error [15]. In the LM-algorithm, the aggregate of the squared error is limited by updating the weights toward the best diminishment of the least square function, if the weights are far from their ideal value [16]. The aggregate of the squared error is limited by expecting the minimum squares function as locally quadratic, and locate the minimum of the quadratic, if the weights are near the ideal value. The output of the MLP-ANN is obtained as

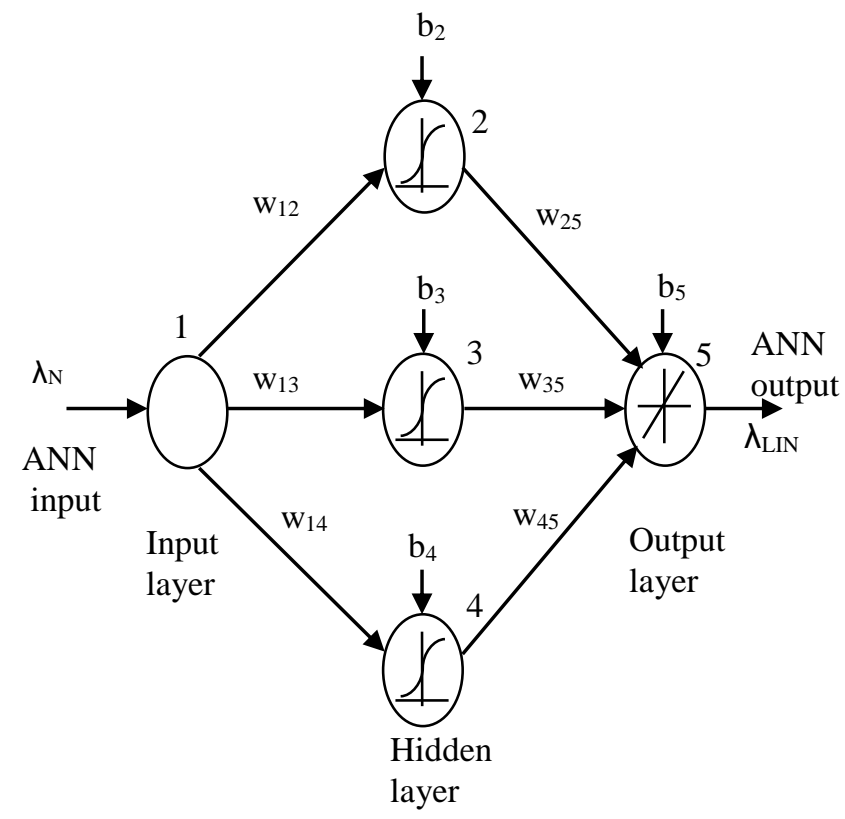

Figure.4 ANN-MLP model $\lambda_{\text {LIN }}=$ purelin $\left\{\begin{array}{c}W_{25} \times\left(\operatorname{losig}\left(W_{12} \times \lambda(T)+b_{2}\right)\right)+ \\ W_{35} \times\left(\operatorname{losig}\left(W_{13} \times \lambda(T)+b_{3}\right)\right)+ \\ W_{45} \times\left(\operatorname{losig}\left(W_{14} \times \lambda(T)+b_{4}\right)\right)+ \\ b_{5}\end{array}\right\}$

The logsig activation function is given by

$$
f(x)=\frac{1}{1+e^{-x}}
$$

\section{Results}

\subsection{Experiment specifications}

A CW laser of peak power $1 \mathrm{mw}$ and central wavelength of $1550 \mathrm{~nm}$ is used in single mode of operation. A single mode fiber optic cable model of length $1 \mathrm{~km}$ and with attenuation factor $0.25 \mathrm{~dB} / \mathrm{km}$ is employed. The fiber Bragg grating refractive index and period has been designed to operate at the central wavelength of $1550 \mathrm{~nm}$ as specified for the $\mathrm{CW}$ laser as well. The specifications of $\mathrm{CW}$ laser, fiber and FBG sensor are shown in Table 2, Table 3 and Table 4 respectively.

\subsection{Simulations and results}

The transmitted and reflected spectrum at temperatures $20^{\circ} \mathrm{C}$ and $320^{\circ} \mathrm{C}$ is shown in Fig. 5 and Fig. 6 respectively.

Table 2. Specifications of CW Laser

\begin{tabular}{|c|c|}
\hline Parameter & Value \\
\hline Peak Power & $1.0 \mathrm{~mW}$ \\
\hline Wavelength & $1550 \mathrm{~nm}$ \\
\hline Mode & Single \\
\hline
\end{tabular}

Table 3. Specifications of Fiber

\begin{tabular}{|c|c|}
\hline Parameter & Value \\
\hline Length & $1000 \mathrm{~m}$ \\
\hline Loss/Attenuation & $0.25 \mathrm{~dB} / \mathrm{km}$ \\
\hline
\end{tabular}

Table 4. Specifications of FBG

\begin{tabular}{|c|c|}
\hline Parameter & Value \\
\hline Period & $0.5457 \mu \mathrm{m}$ \\
\hline Refractive index & 1.42021 \\
\hline Apodisation pattern & Uniform grating \\
\hline Temperature & $0^{\circ} \mathrm{C}-320^{\circ} \mathrm{C}$ \\
\hline
\end{tabular}


On comparing the Fig. 5 and Fig. 6, it can be observed that the peak wavelength has shifted towards the left. This shift however is in the nanoscale and is very minute. Fig. 7 represents the frequency spectrum at $20^{\circ} \mathrm{C}$. The relation between the change in wavelength and temperature is computed for the normal uniform FBG sensor system design implemented in OptSim and is plotted in Fig. 8. Also the temperature vs. wavelength characteristics is plotted in Fig. 9. The temperature wavelength characteristics in Fig. 8 and Fig. 9 indicate a linear relationship, but the sensitivity is drastically reduced. Fig. 10 shows the reflected spectrum for the temperature range $20^{\circ} \mathrm{C}-180^{\circ} \mathrm{C}$ after applying temperature sensitization encapsulation technique in Optisystem and the shift of peak wavelength towards the right can easily be observed. This shift implies that the sensitivity of the system is improved. This improvement is further demonstrated in Fig. 11 where the graph between temperature and wavelength is again plotted with the data obtained after applying temperature sensitization encapsulation technique. For the normal uniform FBG, the temperature sensitivity is $0.375 \mathrm{pm} /{ }^{\circ} \mathrm{C}$. The temperature sensitization encapsulation technique improved temperature sensitivity to $0.0166 \mathrm{~nm} /{ }^{\circ} \mathrm{C}$ which is about 44 times higher than sensitivity of normal uniform FBG, but the linearity error is approximately $\pm 15 \%$.

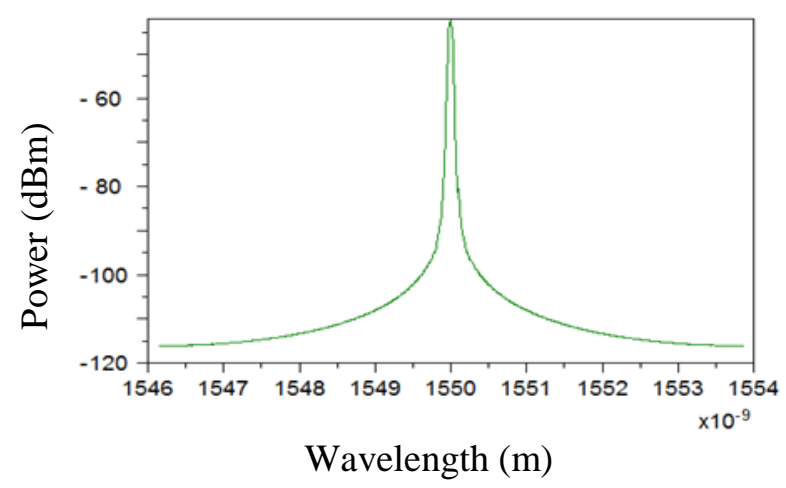

(a)

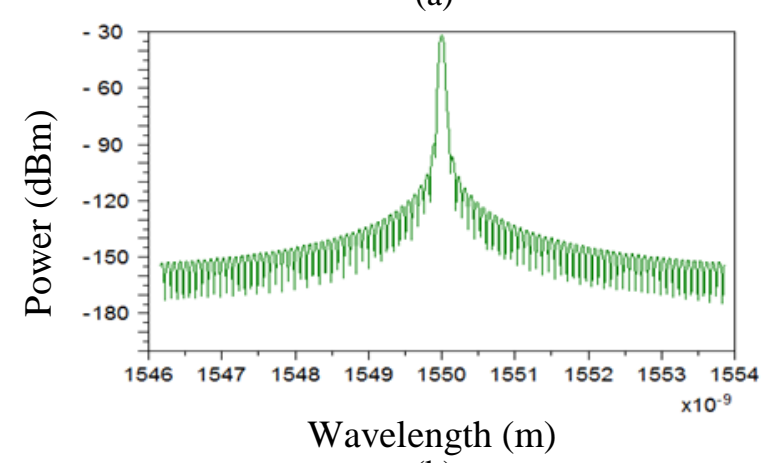

(b)

Figure.5 Wavelength spectrum of normal uniform FBG sensor at $20^{\circ} \mathrm{C}$ : (a) transmitted and (b) reflected

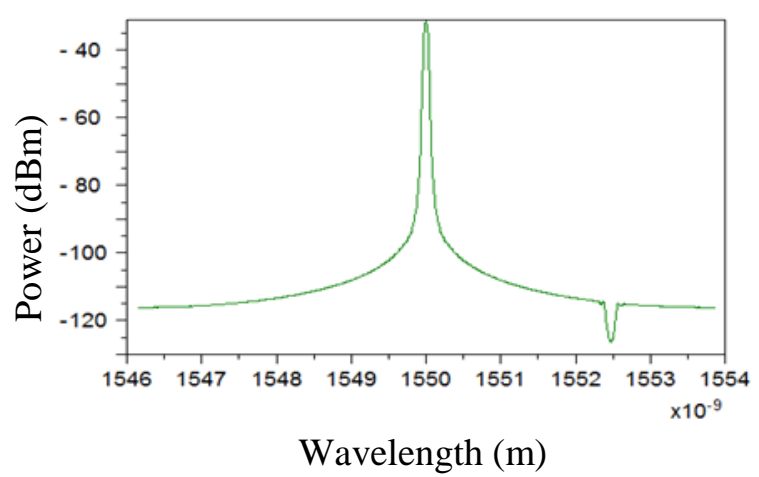

(a)

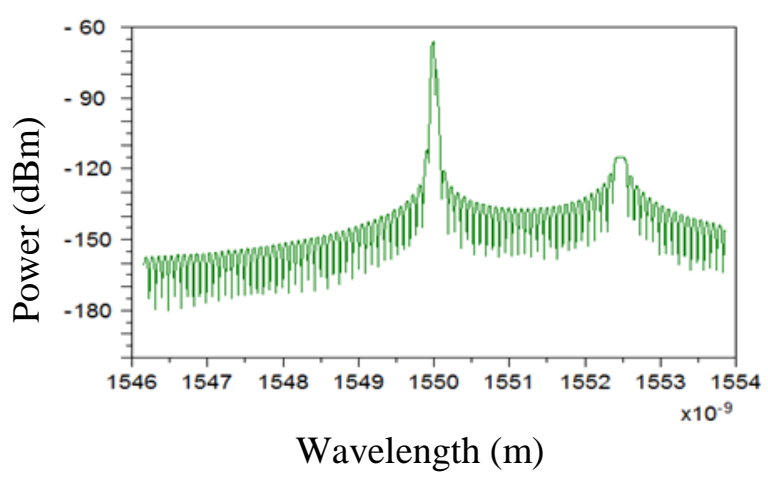

(b)

Figure.6 Wavelength spectrum of normal uniform FBG sensor at $320^{\circ} \mathrm{C}$ : (a) transmitted and (b) reflected

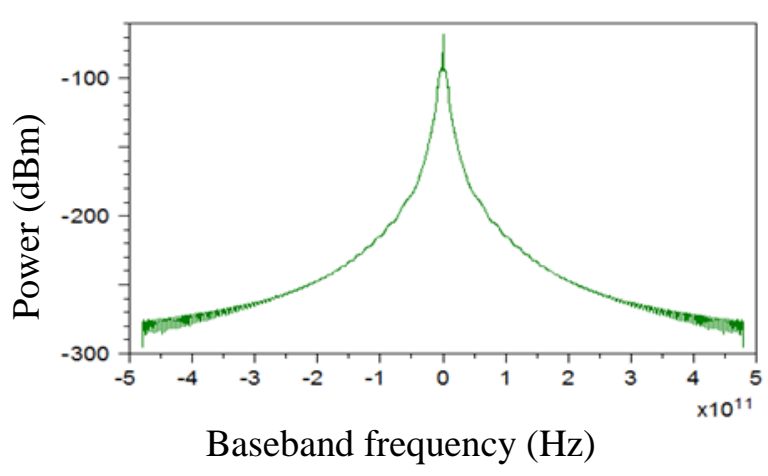

Figure.7 Frequency spectrum of normal uniform FBG sensor at $20^{\circ} \mathrm{C}$

\subsection{ANN training}

The FBG sensor with temperature sensitization encapsulation improves the sensitivity by 44 times, but the linearity error is $\pm 15 \%$ and needs to be reduced. This nonlinear output of the FBG sensor with temperature sensitization encapsulation is applied as the input to the MLP-ANN. The data for the ANN training is selected from the experimental data points in the temperature range of $20^{\circ} \mathrm{C}-180^{\circ} \mathrm{C}$. The output of FBG sensor is normalised in the range $[0,1]$ and applied as input to MLP-ANN. 
The target data for the MLP-ANN is a straight line with a unitary slope. The LM algorithm trains the MLP-ANN and iteratively updates the weights as well as biases. The coding for obtaining the learning and training parameters is carried out in MATLAB.

After the training, testing and validation of the MLP neural network, the MLP-ANN generates the linearized power signal with linearized temperaturewavelength relation. The graphs drawn between the measured temperature and the output wavelength of the MLP-ANN is illustrated in Fig.12, clearly reflects the enhancement of linearity of the FBG sensor without affecting the sensitivity. Another important aspect is that MLP-ANN output closely matches the desired response. The error analysis is depicted in Fig.13. The MLP-ANN technique reduces the linearity error to $\pm 2.8 \%$. The training parameters of the ANN model are given in the Table 5.

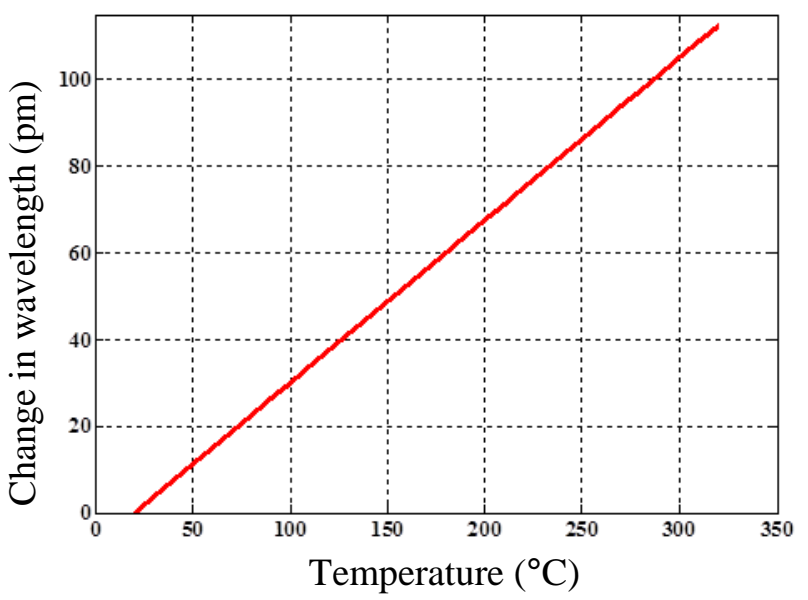

Figure. 8 Change in temperature vs. wavelength characteristics of normal uniform FBG sensor

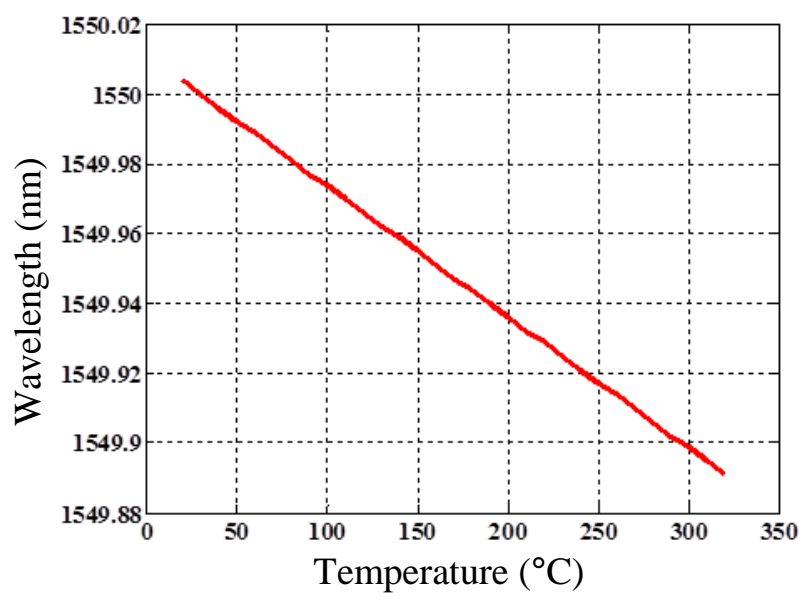

Figure.9 Temperature vs. wavelength characteristics of normal uniform FBG sensor

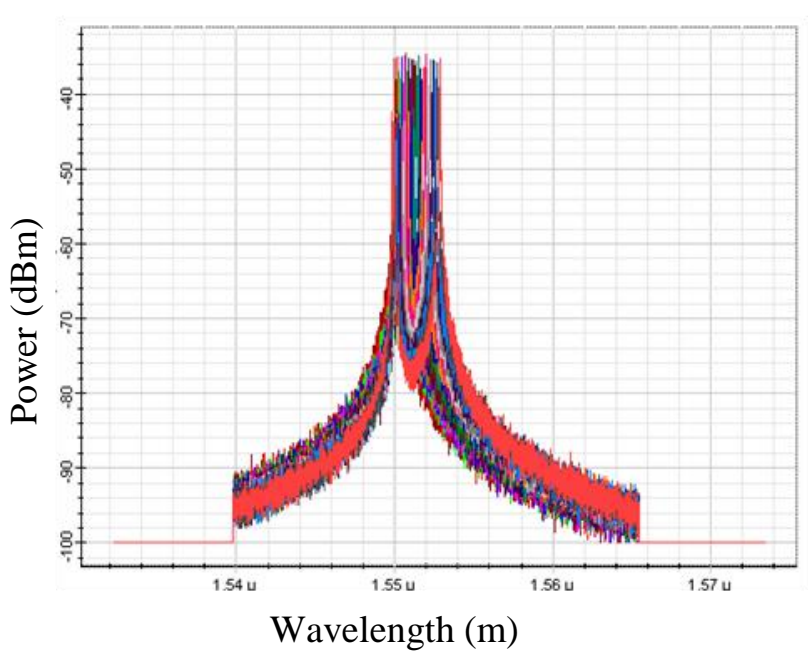

Figure.10 Shift in wavelength of FBG sensor after applying temperature sensitization encapsulation technique

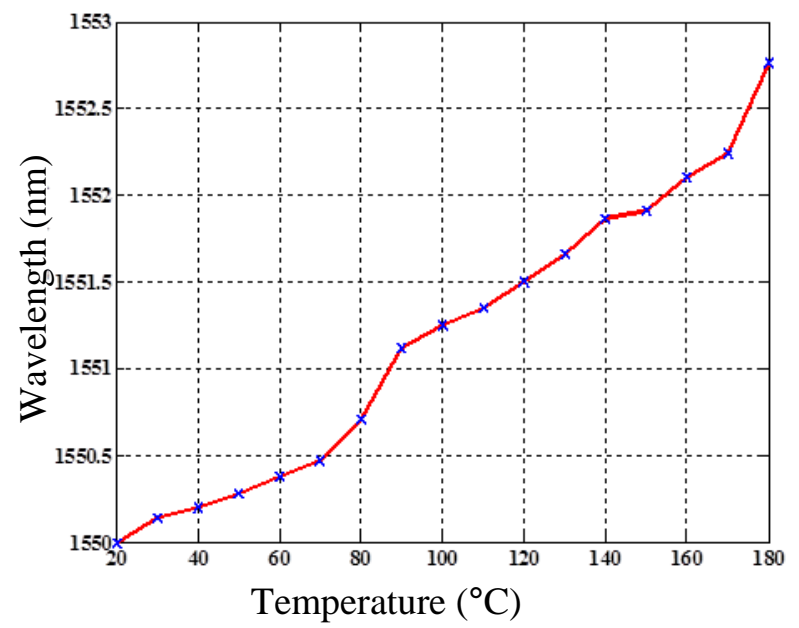

Figure.11 Temperature vs. wavelength characteristics with temperature sensitization encapsulation technique

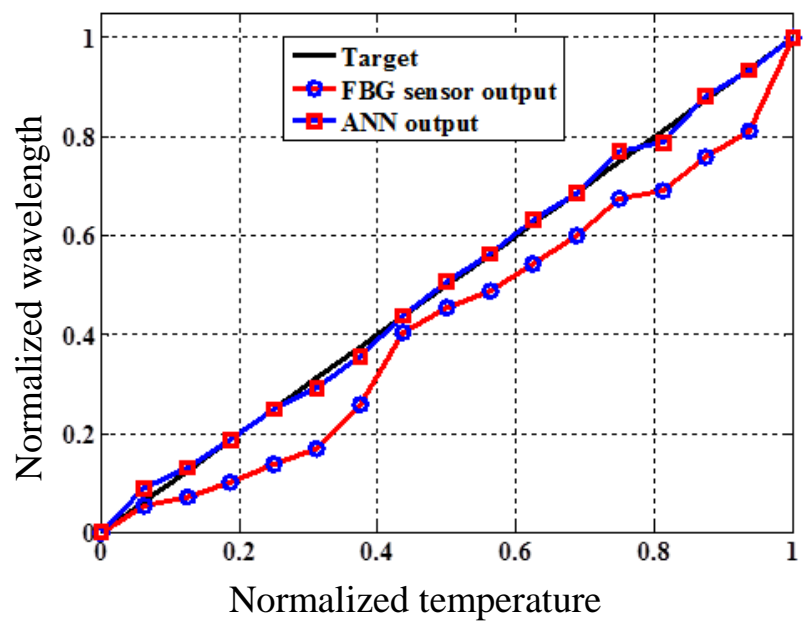

Figure.12 Temperature vs. wavelength characteristics of FBG sensor with sensitization encapsulation and FBG sensor with MLP-ANN 


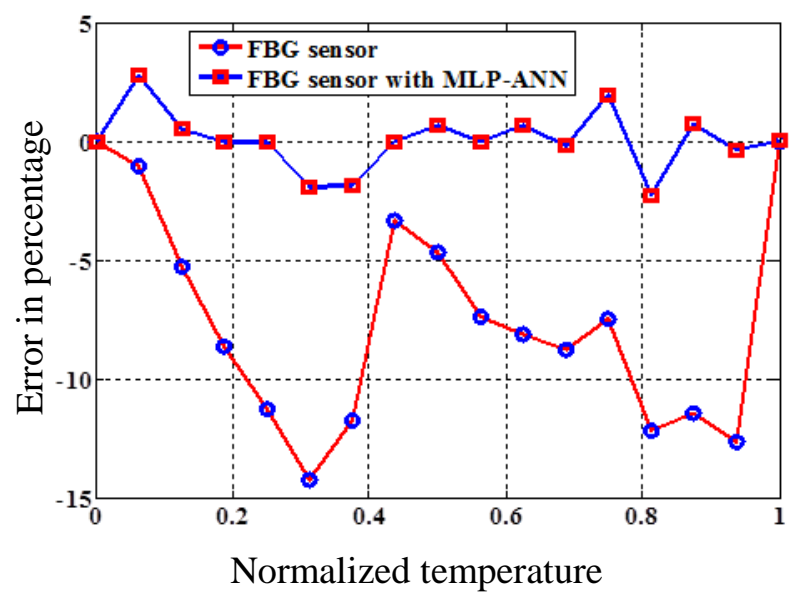

Figure.13 Error analysis of FBG sensor with sensitization encapsulation and FBG sensor with MLP-ANN

Table5. ANN training parameters

\begin{tabular}{|c|c|}
\hline Neural network & $\begin{array}{l}\text { Multilayer feed-forward } \\
\text { neural network }\end{array}$ \\
\hline Number of neurons & $\begin{array}{l}\text { Input neurons: } 1 \\
\text { Hidden neurons: } 3 \\
\text { Output neurons: } 1 \\
\end{array}$ \\
\hline Activation function & $\begin{array}{c}\text { Log sigmoid for hidden layer, } \\
\text { Linear activation function for } \\
\text { output layer }\end{array}$ \\
\hline Training algorithm & Levenberg-Marquardt \\
\hline Weights & $\begin{array}{l}\mathrm{w}_{12}=-3.6095 \\
\mathrm{w}_{13}=-5.6697 \\
\mathrm{w}_{14}=4.0748 \\
\mathrm{w}_{25}=-0.3716 \\
\mathrm{w}_{35}=-0.2583 \\
\mathrm{w}_{45}=0.4920\end{array}$ \\
\hline Biases & $\begin{array}{l}b_{2}=1.5332 \\
b_{3}=-0.4788 \\
b_{4}=-0.4788 \\
b_{5}=-0.1098\end{array}$ \\
\hline
\end{tabular}

\section{Conclusion}

In this paper, a FBG sensor with temperature sensitization encapsulation and MLP-ANN modelling is implemented with high sensitivity and linearity. For the normal uniform FBG, it is observed that by varying the temperature from $20^{\circ} \mathrm{C}$ to $320^{\circ} \mathrm{C}$ in steps of $10^{\circ} \mathrm{C}$, the temperature sensitivity is $0.375 \mathrm{pm} /{ }^{\circ} \mathrm{C}$. The temperature sensitization encapsulation technique improved temperature sensitivity to $0.0166 \mathrm{~nm} /{ }^{\circ} \mathrm{C}$ which is about 44 times higher than sensitivity of normal uniform FBG, but the linearity error is approximately $\pm 15 \%$. To regain the linearity without affecting the sensitivity, MLP-ANN technique is applied and the linearity error is reduced to $\pm 2.8 \%$. The proposed technique provides a precise measurement scheme of temperature over the appreciable range of the FBG sensor. The proposed ANN-based linearization technique may be applied to various other sensors to nullify the adverse effects caused by ambient conditions, aging etc. on their performance. The future scope of the work is to propose a plug in module for embedding the intelligence of ANN and develop a low cost miniature module suitable to internet of things for critical applications of temperature measurement.

\section{References}

[1] M. Yucel and N. F. Ozturk, "Design of a Fiber Bragg rating Multiple Temperature Sensor", In: Proc. of $6^{\text {th }}$ International Conf. On Digital Information and Communication Technology and its Applications (DICTAP), Konya, Turkey, pp.21-23, 2016.

[2] Q. Yu, Y. Zhang, Y. Dong, Y. P. Li, C. Wang, and H. Chen, "Study on Optical Fiber Bragg Grating Temperature Sensors for Human Body Temperature Monitoring", In: Proc. of Symposium on Photonics and Optoelectronics, Shanghai, China, pp.1-4, 2012.

[3] D. Hazarika, K. C. Sarma, and P. K. Sarmah, "Microprocessor Based Temperature Monitoring System Using Optical Fibers", IEEE Sensors Journal, Vol.9, No.9, pp.1025-1028, 2009.

[4] H. M. El-Gammal, H. A. Fayed, A. Abd El-Aziz, and M. H. Aly, "Performance Analysis \& Comparative Study of Uniform, Apodized and Pi-Phase Shifted FBGs for Array of High Performance Temperature Sensors", Optoelectronics and Advanced Materials-Rapid Communications, Vol.9, No.9-10, pp.1251-1259, 2015.

[5] T. Komljenovic, M. Bosiljevac, and Z. Sipus, "Cost Effective FBG based Optical Sensor", In: Proc. of $19^{\text {th }}$ International Conference on Applied Electromagnetics and Communications, Dubrovnik, Croatia, pp.1-4, 2007.

[6] M. Yücel, N.F. Öztürk, M. Yücel, H. H. Göktas and A.E. Gündüz, "Design of a Fiber Bragg Grating Based Temperature Sensor", In: Proc. of $24^{\text {th }}$ Signal Processing and Communication Application Conference (SIU), Zonguldak, Turkey, pp.669-672, 2016.

[7] G. Rajan, Optical Fiber Sensors: Advanced Techniques and Applications, CRC Press, 2015.

[8] H. Heininger, Interrogation of Optical Fiber Sensors for Civil Engineering Applications using Widely Tunable Laser, Ph.d Thesis, Brno University of Technology, 2014. 
[9] M. M. Elgaud, M. S. D. Zan, A. A. G. Abushagur, and A. A. A. Bakar, "Analysis of Independent Strain-Temperature Fiber Bragg Grating Sensing Technique using OptiSystem and OptiGrating", In: Proc. of IEEE 6th International Conference on Photonics (ICP), Kuching, Malaysia, pp.1-3, 2016.

[10]J. Canning, "Fibre Gratings and Devices for Sensors and Lasers", Laser Photonics Review, Vol.2, pp.275-289, 2008.

[11]Z. Tong, J. Zhao, and X. Yang, "Simultaneous Measurement of Axial Strain and Temperature using a PM Fiber Bragg Grating", Microwave Optical Technology Letters, Vol.53, pp.867-870, 2011.

[12]E. Chehura, S. W. James, and R. P. Tatam, "Temperature and Strain Discrimination using a Single Fibre Bragg Grating", Optical Communication, Vol. 275, pp. 344-347, 2007.

[13]T. Erdogan, "Fiber Grating Spectra", IEEE Journal of Lightwave Technology, Vol.15, pp. 1277-1294, 1997.

[14]N. K. Vaegae and V. L. N. Komanapalli, "Development of Thermistor Signal Conditioning Circuit using Artificial Neural Networks", IET Science, Measurement \& Technology, Vol.9, No.8, pp.955-961, 2015.

[15]N. K. Vaegae and V. L. N. Komonapalli, "Development of an ANN-Based Pressure Transducer", IEEE Sensors Journal, Vol.16, No.1, pp.53-60, 2016.

[16]N. K. Vaegae and V. L. N. Komonapalli, "Investigation of Artificial Neural Network Techniques for Thermistor Linearization", Sensors \& Transducers, Vol.186, No.3, pp.55-63, 2015. 